\title{
Synchronization of Two Different Dynamical Systems under Sinusoidal Constraint
}

\author{
Xiaohui Sun ${ }^{1,2}$ and Xilin $\mathrm{Fu}^{1,2}$ \\ ${ }^{1}$ School of Management Science and Engineering, Shandong Normal University, Jinan 250014, China \\ ${ }^{2}$ School of Mathematical Sciences, Shandong Normal University, Jinan 250014, China \\ Correspondence should be addressed to Xilin Fu; xilinfu@gmail.com
}

Received 16 December 2013; Accepted 1 April 2014; Published 29 April 2014

Academic Editor: Qiankun Song

Copyright (C) 2014 X. Sun and X. Fu. This is an open access article distributed under the Creative Commons Attribution License, which permits unrestricted use, distribution, and reproduction in any medium, provided the original work is properly cited.

\begin{abstract}
This paper discusses the synchronization of the Van der Pol equation with a pendulum under the sinusoidal constraint through the theory of discontinuous dynamical systems. The analytical conditions for the sinusoidal synchronization of the Van der Pol equation with a periodically forced pendulum are developed. With the conditions, the sinusoidal synchronizations of the two systems are discussed. Switching points for appearance and vanishing of the partial synchronization are developed.
\end{abstract}

\section{Introduction}

With the development of science and technology, coordinate systems are extensively used to quantitatively describe the characteristics and behaviors of the nature. Through the coordinate systems, one can understand and improve the nature better. In order to research the complexity of the changing process with time, one often uses a known system to compare the unknown process with time. When one obtains the similarity and differences of the two processes for a time interval, the complexity of the unknown dynamical system can be determined through the known one on the similar part of the time interval. The synchronization is a kind of similarity in a time interval, which means that the synchronization is a basis to understand an unknown dynamical system from the well-known one. For the reason above, the synchronization of the dynamical systems is an important concept for dynamical systems.

The investigation on the synchronization goes back to the 17th century. In 1673, Huygens [1] described the synchronization of two pendulum clocks with a weak interaction. After Huygens, many results and progress were achieved [2]. In recent decades, a number of new types of synchronization have appeared, and the four basic synchronizations of dynamical systems are identical synchronization, generalized synchronization, phase synchronization, and anticipated and lag synchronization and amplitude envelope synchronization. For any synchronization, there is at least one constraint, and such synchronization may experience the asymptotic stability characteristics. This issue can be referred to in Boccaletti [3] and Pikovsky et al. [4].

In 1990, Pecora and Carroll [5] studied the identical synchronization of two systems connected with common signals by using the criterion of the sub-Lyapunov exponents. In the problem, the signals are treated as constraints for the two systems. Carroll and Pecora [6] used the synchronized circuits to simulate the synchronization of chaos. Since then, such efforts induced a lot of attention to developing the control methods and schemes of the synchronization with constraints. In 1992, two methods for chaos control to achieve the synchronization of two chaotic systems were presented by Pyragas [7] with a small time continuous perturbation. On the basis above, Kapitaniak [8] presented the synchronization of two chaotic systems with such methods in 1994. In the same year, Ding and Ott [9] pointed out that the slave system is not necessary to be a replica of part of master systems. Under the directionally coupled constraint, the generalized synchronization of chaos was discussed by Rulkov et al. [10] in 1995. Kocarev and Parlitz [11] presented the idea that the given systems were treated as the active and passive systems. In 1996, Pyragas [12] discussed the weak and strong synchronization of chaos. In 1997, Ding et al. [13] gave a 
review on the chaotic control and synchronization, and an adaptive synchronization of chaos was presented by Boccaletti. In 2004, Campos et al. [14] described the multimodal synchronization with chaos, and the definition of masterslave synchronization was presented. In 2006, Teufel et al. [15] discussed the synchronization of two flow-excited pendula, and a review on stability of synchronic dynamics was presented by Chen et al. [16]. In 2007 and 2009, Chen discussed the complete and generalized synchronizations of the systems under noise perturbations $[17,18]$.

From the above discussion of the synchronization, the synchronization of dynamical systems is that the corresponding flows of the dynamical systems are constrained under special constraint for a time interval. When the constraints are treated as constraint boundaries, the theory of discontinuous dynamical systems can be used to the synchronization of dynamical systems. And the form of synchronization is different when the constraints are different. In 2005, Luo [19] developed a theory for discontinuous dynamical systems and got a lot of results [20-24]. In this paper, we will discuss how the Van der Pol equation will be synchronized with a periodically forced pendulum under the sinusoidal constraint. Consider the pendulum to be the master system and the Van der Pol equation to be the slave system. Under the sinusoidal constraint, how the slave system will be synchronized with the master system is investigated. The analytical conditions of the synchronization will be developed.

\section{Master and Slave Systems}

Consider a periodically exited pendulum as a master system:

$$
\ddot{x}+a_{0} \sin x=A_{0} \cos \omega t .
$$

Consider the Van der Pol equation as a slave system:

$$
\ddot{y}+\varepsilon\left(y^{2}-1\right) \dot{y}+y=0, \quad \varepsilon>0 .
$$

For convenience, the state variables are defined as

$$
X=\left(x_{1}, x_{2}\right)^{T}, \quad Y=\left(y_{1}, y_{2}\right)^{T},
$$

and the vector fields are defined as

$$
\mathscr{F}(X, t)=\left(x_{2}, \mathscr{F}_{2}(X, t)\right), \quad F(Y, t)=\left(y_{2}, F_{2}(Y, t)\right) .
$$

Thus the master system is in the form

$$
\dot{X}=\mathscr{F}(X, t),
$$

where

$$
\dot{x}_{1} \equiv x_{2}, \quad \dot{x}_{2}=\mathscr{F}_{2}(X, t)=-a_{0} \sin x_{1}+A_{0} \cos \omega t .
$$

The slave system becomes

$$
\dot{Y}=F(Y, t),
$$

where

$$
\dot{y}_{1} \equiv y_{2}, \quad \dot{y}_{2}=F_{2}(Y, t)=-\varepsilon\left(y_{1}^{2}-1\right) y_{2}-y_{1} .
$$

Consider the slave system synchronizing with the master system with certain function constraint

$$
\Phi(X, Y, \lambda)=0
$$

The identical synchronization can be as a special case $(\Phi=X-Y=0)$. To get the synchronization, the constraint should be inserted:

$$
\varphi_{1}=y_{1}-\sin x_{1}=0, \quad \varphi_{2}=y_{2}-x_{2} \cos x_{1} .
$$

Consider the master system to be independent. With a control law, the slave system is discontinuous and becomes

$$
\dot{Y}=F(Y, t)+U(X, Y, t)
$$

where

$$
\begin{gathered}
U(X, Y, t)=\left(u_{1}, u_{2}\right)^{T}, \quad u_{1}=-k_{1} \operatorname{sgn}\left(y_{1}-\sin x_{1}\right), \\
u_{2}=-k_{2} \operatorname{sgn}\left(y_{2}-x_{2} \cos x_{1}\right), \\
F(Y, t)+U(X, Y, t)=\left(f_{1}, f_{2}\right)^{T} .
\end{gathered}
$$

The master system is independent of the slave system, and the flow will not be changed. But the slave system will be controlled by the master system to be synchronized. Under the control, the slave system possesses four regions and will be discontinuous. The controlled slave system becomes

(i) for $y_{1}>\sin x_{1}$ and $y_{2}>x_{2} \cos x_{1}$,

$$
\begin{gathered}
f_{1}(Y, t)=y_{2}-k_{1}, \\
f_{2}(Y, t)=-\varepsilon\left(y_{1}^{2}-1\right) y_{2}-y_{1}-k_{2} ;
\end{gathered}
$$

(ii) for $y_{1}>\sin x_{1}$ and $y_{2}<x_{2} \cos x_{1}$,

$$
\begin{gathered}
f_{1}(Y, t)=y_{2}-k_{1}, \\
f_{2}(Y, t)=-\varepsilon\left(y_{1}^{2}-1\right) y_{2}-y_{1}+k_{2} ;
\end{gathered}
$$

(iii) for $y_{1}<\sin x_{1}$ and $y_{2}<x_{2} \cos x_{1}$,

$$
\begin{gathered}
f_{1}(Y, t)=y_{2}+k_{1}, \\
f_{2}(Y, t)=-\varepsilon\left(y_{1}^{2}-1\right) y_{2}-y_{1}+k_{2} ;
\end{gathered}
$$

(iv) for $y_{1}<\sin x_{1}$ and $y_{2}>x_{2} \cos x_{1}$,

$$
\begin{gathered}
f_{1}(Y, t)=y_{2}+k_{1}, \\
f_{2}(Y, t)=-\varepsilon\left(y_{1}^{2}-1\right) y_{2}-y_{1}-k_{2} .
\end{gathered}
$$

\section{Discontinuous Description}

Under the control laws, the Van der Pol equation has four regions with different vector fields, four boundaries with four different vector fields, and an intersection point with one vector field. The intersection point is the synchronization of 
the Van der Pol equation with the pendulum. Four domains $\Omega_{\alpha}(\alpha=1,2,3,4)$ of the Van der Pol equation in phase space are defined as

$$
\begin{aligned}
& \Omega_{1}=\left\{\left(y_{1}, y_{2}\right) \mid y_{1}-\sin x_{1}(t)>0, y_{2}-x_{2}(t) \cos x_{1}>0\right\}, \\
& \Omega_{2}=\left\{\left(y_{1}, y_{2}\right) \mid y_{1}-\sin x_{1}(t)>0, y_{2}-x_{2}(t) \cos x_{1}<0\right\}, \\
& \Omega_{3}=\left\{\left(y_{1}, y_{2}\right) \mid y_{1}-\sin x_{1}(t)<0, y_{2}-x_{2}(t) \cos x_{1}<0\right\}, \\
& \Omega_{4}=\left\{\left(y_{1}, y_{2}\right) \mid y_{1}-\sin x_{1}(t)<0, y_{2}-x_{2}(t) \cos x_{1}>0\right\} .
\end{aligned}
$$

The corresponding boundaries are defined as

$$
\begin{aligned}
& \partial \Omega_{12}=\left\{\left(y_{1}, y_{2}\right) \mid y_{1}-\sin x_{1}>0, y_{2}-x_{2}(t) \cos x_{1}=0\right\}, \\
& \partial \Omega_{23}=\left\{\left(y_{1}, y_{2}\right) \mid y_{1}-\sin x_{1}=0, y_{2}-x_{2}(t) \cos x_{1}<0\right\}, \\
& \partial \Omega_{34}=\left\{\left(y_{1}, y_{2}\right) \mid y_{1}-\sin x_{1}<0, y_{2}-x_{2}(t) \cos x_{1}=0\right\}, \\
& \partial \Omega_{14}=\left\{\left(y_{1}, y_{2}\right) \mid y_{1}-\sin x_{1}=0, y_{2}-x_{2}(t) \cos x_{1}>0\right\} .
\end{aligned}
$$

The intersection point of the boundaries $\partial \Omega_{\alpha \beta}(\alpha, \beta=$ $1,2,3,4 ; \alpha \neq \beta)$ in phase space is

$$
\begin{aligned}
\angle \partial \Omega_{\alpha \beta} & =\bigcap_{\alpha, \beta=1}^{4} \partial \Omega_{\alpha \beta} \\
& =\left\{\left(y_{1}, y_{2}\right) \mid y_{2}-x_{2} \cos x_{1}=0, y_{1}-\sin x_{1}=0\right\}
\end{aligned}
$$

Similar to the usual illustration in the discontinuous dynamical systems, the subdomains and boundaries are illustrated in Figures 1 and 2.

The corresponding domains and boundaries are labeled, and the dashed curves give the two boundaries. The two boundaries of the controlled Van der Pol equation are determined by the displacement and velocity of the pendulum. The intersection point of the two boundaries is labeled by a filled circular symbol.

Based on the previously defined $\Omega_{\alpha}$, the corresponding dynamical system of the controlled slave system is defined as

$$
\dot{Y}^{(\alpha)}=F^{(\alpha)}\left(Y^{(\alpha)}, t\right)
$$

where

$$
\begin{gathered}
f_{1}^{(\alpha)}\left(Y^{(\alpha)}, t\right)=y_{2}^{(\alpha)}-k_{1}, \quad \text { for } \alpha=1,2, \\
f_{1}^{(\alpha)}\left(Y^{(\alpha)}, t\right)=y_{2}^{(\alpha)}+k_{1}, \quad \text { for } \alpha=3,4, \\
f_{2}^{(\alpha)}\left(Y^{(\alpha)}, t\right)=-\varepsilon\left(y_{1}^{2}-1\right) y_{2}-y_{1}-k_{2}, \quad \text { for } \alpha=1,4, \\
f_{2}^{(\alpha)}\left(Y^{(\alpha)}, t\right)=-\varepsilon\left(y_{1}^{2}-1\right) y_{2}-y_{1}+k_{2}, \quad \text { for } \alpha=2,3 .
\end{gathered}
$$

The boundary flow is controlled by the master system, and the boundaries change with times. The corresponding dynamical systems on the boundaries are

$$
\begin{gathered}
\dot{Y}^{(\alpha, \beta)}=F^{(\alpha, \beta)}\left(Y^{(\alpha, \beta)}, X(t), t\right), \\
\dot{X}=\mathscr{F}(X, t),
\end{gathered}
$$

where $f_{1}^{(\alpha, \beta)}\left(Y^{(\alpha, \beta)}, X(t), t\right)=y_{2}^{(\alpha \beta)}(t)=x_{2}(t) \cos x_{1}$ and $f_{2}^{(\alpha, \beta)}\left(Y^{(\alpha, \beta)}, X(t), t\right)=\dot{x}_{2} \cos x_{1}-x_{2}^{2} \sin x_{1}$, with

$$
\begin{array}{r}
y_{1}^{(\alpha, \beta)}=\sin x_{1}(t), \quad y_{2}^{(\alpha, \beta)}=x_{2}(t) \cos x_{1} \\
\text { on } \partial \Omega_{\alpha \beta} \text { for }(\alpha, \beta)=(2,3),(1,4), \\
y_{1}^{(\alpha, \beta)}=\sin x_{1}(t)+c, \quad y_{2}^{(\alpha, \beta)}=x_{2}(t) \cos x_{1} \\
\text { on } \partial \Omega_{\alpha \beta} \text { for }(\alpha, \beta)=(1,2),(3,4) .
\end{array}
$$

From the above equation, it can be seen that the flow is controlled by the master system on the boundaries, and that the boundaries change with time. From the systems in the absolute coordinate, it is difficult to develop the analytical conditions. Thus, the relative coordinates are defined as

$$
z_{1}=y_{1}-\sin x_{1}, \quad \dot{z}_{1} \equiv z_{2}=y_{2}-x_{2} \cos x_{1} .
$$
become

The domain and boundaries in the relative coordinate

$$
\begin{gathered}
\Omega_{1}(t)=\left\{\left(z_{1}, z_{2}\right) \mid z_{1}>0, z_{2}>0\right\}, \\
\Omega_{2}(t)=\left\{\left(z_{1}, z_{2}\right) \mid z_{1}>0, z_{2}<0\right\}, \\
\Omega_{3}(t)=\left\{\left(z_{1}, z_{2}\right) \mid z_{1}<0, z_{2}<0\right\}, \\
\Omega_{4}(t)=\left\{\left(z_{1}, z_{2}\right) \mid z_{1}<0, z_{2}>0\right\}, \\
\partial \Omega_{12}(t)=\left\{\left(z_{1}, z_{2}\right) \mid z_{2}=0\right\}=\partial \Omega_{34}(t), \\
\partial \Omega_{23}(t)=\left\{\left(z_{1}, z_{2}\right) \mid z_{1}=0\right\}=\partial \Omega_{14}(t) . \\
\angle \partial \Omega_{\alpha \beta}=\bigcap_{\alpha, \beta=1}^{4} \partial \Omega_{\alpha \beta}=\left\{\left(z_{1}, z_{2}\right) \mid z_{1}=0, z_{2}=0\right\} .
\end{gathered}
$$

The subdomains and boundaries in the relative coordinates are illustrated in Figure 3.

The velocity and displacement boundaries in the relative coordinates are constant.

The controlled slave system in relative coordinates becomes

$$
\begin{aligned}
\dot{Z}^{(\alpha)} & =G^{(\alpha)}\left(Z^{(\alpha)}, X, t\right), \\
\dot{X} & =\mathscr{F}(X, t),
\end{aligned}
$$




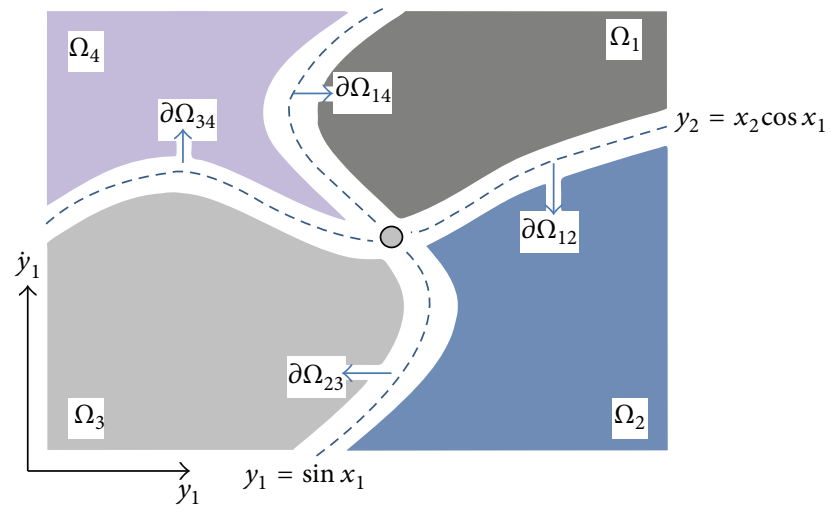

FIGURE 1: Subdomains and boundaries of controlled slave system in absolute coordinates.

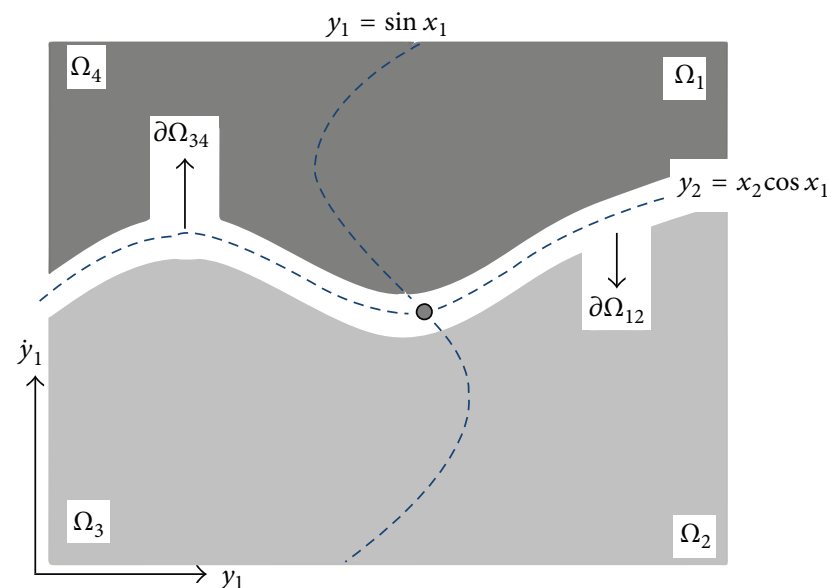

(a) Velocity boundary

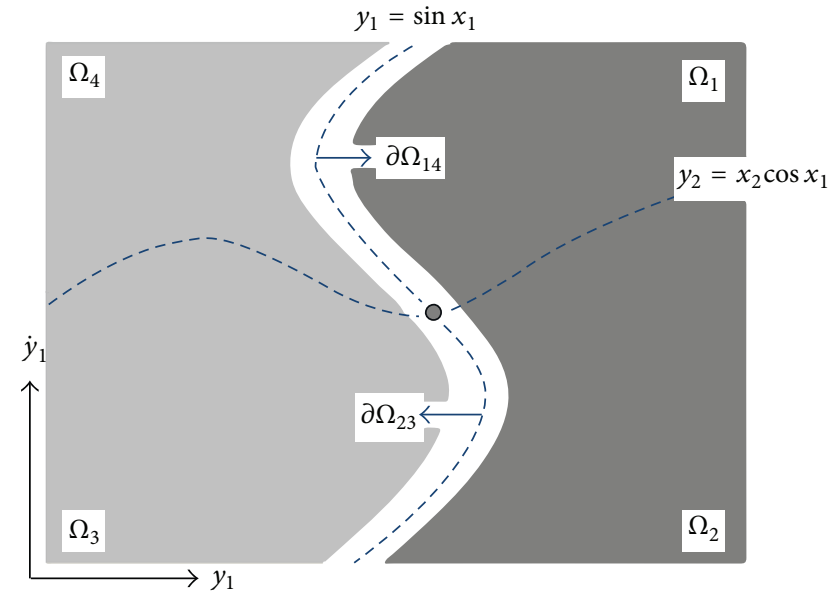

(b) Displacement boundary

FIgURE 2: Separated illustrations for the two boundaries.

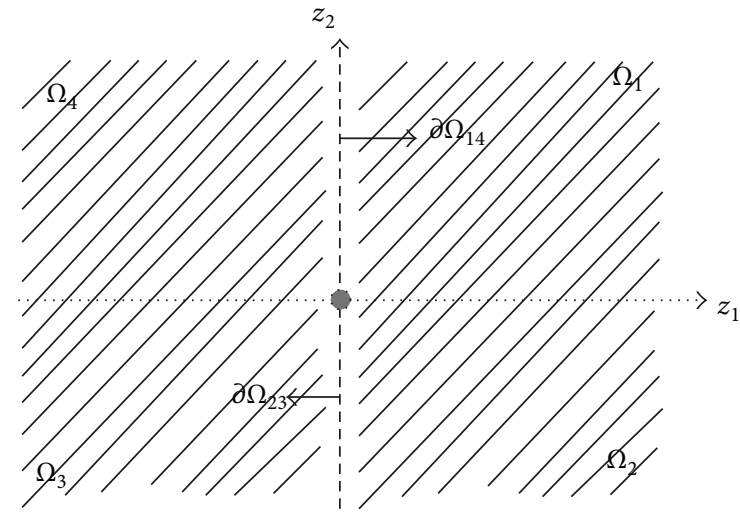

(a) Relative velocity boundary

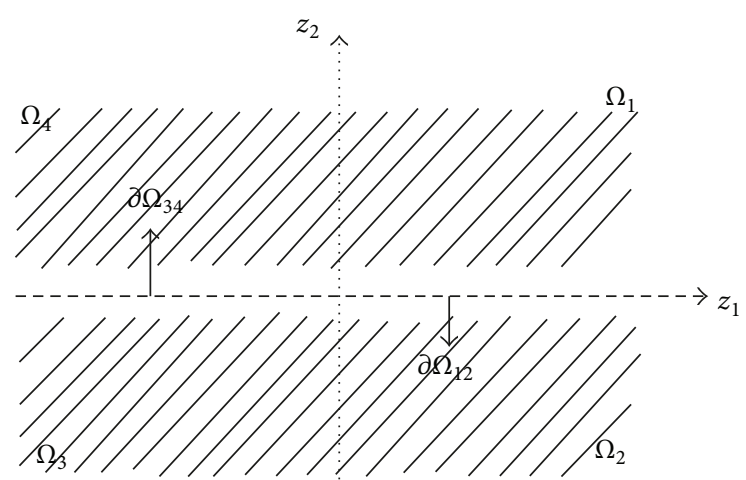

(b) Relative displacement boundary

FIGURE 3: Separated illustrations for the two boundaries in the relative coordinate. 
where

$$
\begin{gathered}
g_{1}^{(\alpha)}\left(Z^{(\alpha)}, X, t\right)=z_{2}^{(\alpha)}-k_{1} \quad \text { for } \alpha=1,2, \\
g_{1}^{(\alpha)}\left(Z^{(\alpha)}, X, t\right)=z_{2}^{(\alpha)}+k_{1} \quad \text { for } \alpha=3,4, \\
g_{2}^{(\alpha)}\left(Z^{(\alpha)}, X, t\right)=\mathscr{G}\left(Z^{(\alpha)}, X, t\right)-k_{2} \quad \text { for } \alpha=1,4, \\
g_{2}^{(\alpha)}\left(Z^{(\alpha)}, X, t\right)=\mathscr{G}\left(Z^{(\alpha)}, X, t\right)+k_{2} \quad \text { for } \alpha=2,3,
\end{gathered}
$$

with

$$
\begin{aligned}
\mathscr{G}\left(Z^{(\alpha)}, X, t\right)= & \dot{y}_{2}-\dot{x}_{2} \cos x_{1}+x_{2}^{2} \sin x_{1} \\
= & -\varepsilon\left(y_{1}^{2}-1\right) y_{2}-y_{1}+a_{0} \sin x_{1} \cos x_{1} \\
& -A_{0} \cos \omega t \cos x_{1}+x_{2}^{2} \sin x_{1} \\
= & -\varepsilon\left[\left(\sin x_{1}+z_{1}\right)^{2}-1\right]\left(x_{2} \cos x_{1}+z_{2}\right) \\
& -\left(\sin x_{1}+z_{1}\right)+a_{0} \sin x_{1} \cos x_{1} \\
& -A_{0} \cos \omega t \cos x_{1}+x_{2}^{2} \sin x_{1} .
\end{aligned}
$$

The dynamics on the boundary can be written as

$$
\begin{gathered}
\dot{Z}^{(\alpha \beta)}=G^{(\alpha \beta)}\left(Z^{(\alpha \beta)}, X, t\right), \\
\dot{X}=\mathscr{F}(X, t),
\end{gathered}
$$

where

$$
g_{1}^{(\alpha \beta)}\left(Z^{(\alpha \beta)}, X, t\right)=z_{2}=0, \quad g_{2}^{(\alpha \beta)}\left(Z^{(\alpha \beta)}, X, t\right)=0,
$$

with

$z_{1}^{(\alpha \beta)}=0, \quad z_{2}^{(\alpha \beta)}=0 \quad$ on $\partial \Omega_{\alpha \beta}$ for $(\alpha, \beta)=(2,3),(1,4)$,

$z_{1}^{(\alpha \beta)}=c, \quad z_{2}^{(\alpha \beta)}=0 \quad$ on $\partial \Omega_{\alpha \beta}$ for $(\alpha, \beta)=(1,2),(3,4)$

\section{Analytical Conditions for Synchronization}

The synchronization of the two systems under the sinusoidal constraint will be discussed. The $G$-functions are introduced in the relative coordinates for $Z_{m} \in \partial \Omega_{i j}$ at $t=t_{m}$ :

$$
\begin{aligned}
& G_{\partial \Omega_{i j}}^{(\alpha)}\left(Z_{m}, X, t_{m \pm}\right)=\vec{n}_{\partial \Omega_{i j}}^{T} \cdot\left[G^{(\alpha)}\left(Z_{m}, X, t_{m \pm}\right)\right.\left.-G^{(i j)}\left(Z_{m}, X, t_{m \pm}\right)\right], \\
& G_{\partial \Omega_{i j}}^{(1, \alpha)}\left(Z_{m}, X, t_{m \pm}\right)=\vec{n}_{\partial \Omega_{i j}}^{T} \cdot\left[D G^{(\alpha)}\left(Z_{m}, X, t_{m \pm}\right)\right. \\
&\left.-D G^{(i j)}\left(Z_{m}, X, t_{m \pm}\right)\right],
\end{aligned}
$$

where $G_{\partial \Omega_{i j}}^{(\alpha)}\left(Z_{m}, X, t_{m \pm}\right)$ and $G_{\partial \Omega_{i j}}^{(1, \alpha)}\left(Z_{m}, X, t_{m \pm}\right)$ are the zeroorder and first-order $G$-functions of the flow in the domain $\Omega_{\alpha}(\alpha \in\{i, j\})$ at the boundary $\partial \Omega_{i j},(i, j) \in$ $\{(1,2),(2,3),(3,4),(1,4)\}$. In this paper, the normal vectors of the boundaries are

$$
\vec{n}_{\partial \Omega_{12}}=\vec{n}_{\partial \Omega_{34}}=(0,1)^{T}, \quad \vec{n}_{\partial \Omega_{23}}=\vec{n}_{\partial \Omega_{14}}=(1,0)^{T} .
$$

The corresponding $G$-functions for the boundary are

$$
\begin{aligned}
& G_{\partial \Omega_{12}}^{(\alpha)}\left(Z_{m}, X, t_{m \pm}\right)=G_{\partial \Omega_{34}}^{(\alpha)}\left(Z_{m}, X, t_{m \pm}\right)=g_{2}^{(\alpha)}\left(Z_{m}, X, t_{m \pm}\right) \\
& G_{\partial \Omega_{23}}^{(\alpha)}\left(Z_{m}, X, t_{m \pm}\right)=G_{\partial \Omega_{14}}^{(\alpha)}\left(Z_{m}, X, t_{m \pm}\right)=g_{1}^{(\alpha)}\left(Z_{m}, X, t_{m \pm}\right) \\
& G_{\partial \Omega_{12}}^{(1, \alpha)}\left(Z_{m}, X, t_{m \pm}\right)=G_{\partial \Omega_{34}}^{(1, \alpha)}\left(Z_{m}, X, t_{m \pm}\right) \\
&=D g_{2}^{(\alpha)}\left(Z_{m}, X, t_{m \pm}\right) \\
& G_{\partial \Omega_{23}}^{(1, \alpha)}\left(Z_{m}, X, t_{m \pm}\right)=G_{\partial \Omega_{14}}^{(1, \alpha)}\left(Z_{m}, X, t_{m \pm}\right) \\
&=D g_{1}^{(\alpha)}\left(Z_{m}, X, t_{m \pm}\right)
\end{aligned}
$$

where

$$
\begin{aligned}
D g_{1}^{(\alpha)}\left(Z^{(\alpha)}, X, t\right)= & g_{2}^{(\alpha)}\left(Z^{(\alpha)}, X, t\right), \quad \alpha=1,2,3,4 \\
D g_{2}^{(\alpha)}\left(Z^{(\alpha)}, X, t\right)= & D \mathscr{G}\left(Z^{(\alpha)}, X, t\right) \\
= & \left(-2 \varepsilon y_{1}-1\right) y_{2}+\varepsilon^{2}\left(y_{1}^{2}-1\right)^{2} \\
& +\varepsilon\left(y_{1}^{2}-1\right) y_{1} \\
& +\left(a_{0} x_{2} \cos x_{1}+A_{0} \omega \sin \omega t\right. \\
& \left.\quad+x_{2}^{3}\right) \cos x_{1} \\
& +3\left(A_{0} \cos \omega t-a_{0} \sin x_{1}\right) x_{2} \sin x_{1} .
\end{aligned}
$$

\subsection{Flow Switchability on the Separation Boundary}

(i) A flow sliding on the boundaries of $\partial \Omega_{12}, \partial \Omega_{34}, \partial \Omega_{23}$, and $\partial \Omega_{14}$ for the controlled system satisfies

$$
\begin{array}{r}
G_{\partial \Omega_{12}}^{(1)}\left(Z_{m}^{(1)}, X, t_{m-}\right)=g_{2}^{(1)}\left(Z_{m}, X, t_{m-}\right)<0 \\
G_{\partial \Omega_{12}\left(Z_{m}^{(2)}, X, t_{m-}\right)=} g_{2}^{(2)}\left(Z_{m}, X, t_{m-}\right)>0 \\
\quad \text { for } Z_{m} \in \partial \Omega_{12}, \\
\left.G_{\partial \Omega_{34}\left(Z_{m}\right)}^{(3)}, X, t_{m-}\right)=g_{2}^{(3)}\left(Z_{m}, X, t_{m-}\right)>0 \\
G_{\partial \Omega_{34}\left(Z_{m}\right)}^{(3)}\left(Z_{m-}^{(4)}, X, t_{m-}\right)=g_{2}^{(4)}\left(Z_{m}, X, t_{m-}\right)<0 \\
\quad \text { for } Z_{m} \in \partial \Omega_{34}, \\
G_{\partial \Omega_{23}(2)}^{(2)}\left(Z_{m}^{(2)}, X, t_{m-}\right)=g_{1}^{(2)}\left(Z_{m}, X, t_{m-}\right)<0 \\
G_{\partial \Omega_{23}(3)}^{(3)}\left(Z_{m}^{(3)}, X, t_{m-}\right)=g_{1}^{(3)}\left(Z_{m}, X, t_{m-}\right)>0 \\
\quad \text { for } Z_{m} \in \partial \Omega_{23},
\end{array}
$$




$$
\begin{aligned}
& G_{\partial \Omega_{14}}^{(1)}\left(Z_{m}^{(1)}, X, t_{m-}\right)=g_{1}^{(1)}\left(Z_{m}, X, t_{m-}\right)<0 \\
& G_{\partial \Omega_{14}}^{(4)}\left(Z_{m}^{(4)}, X, t_{m-}\right)=g_{1}^{(4)}\left(Z_{m}, X, t_{m-}\right)>0
\end{aligned}
$$$$
\text { for } Z_{m} \in \partial \Omega_{14} \text {. }
$$

(ii) A flow passing through the boundaries of $\partial \Omega_{12}, \partial \Omega_{34}, \partial \Omega_{23}$, and $\partial \Omega_{14}$ for the controlled system satisfies

$$
\begin{aligned}
& G_{\partial \Omega_{12}}^{(1)}\left(Z_{m}^{(1)}, X, t_{m-}\right)=g_{2}^{(1)}\left(Z_{m}, X, t_{m-}\right)<0 \\
& G_{\partial \Omega_{12}}^{(2)}\left(Z_{m}^{(2)}, X, t_{m+}\right)=g_{2}^{(2)}\left(Z_{m}, X, t_{m+}\right)<0 \\
& \text { from } \Omega_{1} \text { to } \Omega_{2} \text {, } \\
& G_{\partial \Omega_{34}}^{(3)}\left(Z_{m}^{(3)}, X, t_{m-}\right)=g_{2}^{(3)}\left(Z_{m}, X, t_{m-}\right)>0 \\
& G_{\partial \Omega_{34}}^{(4)}\left(Z_{m}^{(4)}, X, t_{m+}\right)=g_{2}^{(4)}\left(Z_{m}, X, t_{m+}\right)>0 \\
& \text { from } \Omega_{3} \text { to } \Omega_{4}, \\
& G_{\partial \Omega_{23}}^{(2)}\left(Z_{m}^{(2)}, X, t_{m-}\right)=g_{1}^{(2)}\left(Z_{m}, X, t_{m-}\right)<0 \\
& G_{\partial \Omega_{23}}^{(3)}\left(Z_{m}^{(3)}, X, t_{m+}\right)=g_{1}^{(3)}\left(Z_{m}, X, t_{m+}\right)<0 \\
& \text { from } \Omega_{2} \text { to } \Omega_{3} \text {, } \\
& G_{\partial \Omega_{14}}^{(4)}\left(Z_{m}^{(4)}, X, t_{m-}\right)=g_{1}^{(4)}\left(Z_{m}, X, t_{m-}\right)>0 \\
& G_{\partial \Omega_{14}}^{(1)}\left(Z_{m}^{(1)}, X, t_{m+}\right)=g_{1}^{(1)}\left(Z_{m}, X, t_{m+}\right)>0
\end{aligned}
$$

from $\Omega_{4}$ to $\Omega_{1}$.

(iii) A flow grazing the boundaries of $\partial \Omega_{12}, \partial \Omega_{34}, \partial \Omega_{23}$, and $\partial \Omega_{14}$ for the controlled system satisfies

$$
\begin{gathered}
G_{\partial \Omega_{12}}^{(0, \alpha)}\left(Z_{m}, X, t_{m \pm}\right)=g_{2}^{(\alpha)}\left(Z_{m}, X, t_{m \pm}\right)=0 \\
(-1)^{\alpha} G_{\partial \Omega_{12}}^{(1, \alpha)}\left(Z_{m}, X, t_{m \pm}\right)=(-1)^{\alpha} D g_{2}^{(\alpha)}\left(Z_{m}, X, t_{m \pm}\right)<0 \\
\text { for } Z_{m} \in \partial \Omega_{12} \text { in } \Omega_{\alpha}(\alpha \in\{1,2\}), \\
(-1)^{\alpha} G_{\partial \Omega_{34}}^{(1, \alpha)}\left(Z_{m}, X, t_{m \pm}\right)=(-1)^{\alpha} D g_{2}^{(\alpha)}\left(Z_{m}, X, t_{m \pm}\right)>0 \\
G_{\partial \Omega_{34}}^{(0, \alpha)}\left(Z_{m}, X, t_{m \pm}\right)=g_{2}^{(\alpha)}\left(Z_{m}, X, t_{m \pm}\right)=0 \\
G_{\partial \Omega_{23}}^{(0, \alpha)}\left(Z_{m}, X, t_{m \pm}\right)=g_{1}^{(\alpha)}\left(Z_{m}, X, t_{m \pm}\right)=0 \\
(-1)^{\alpha} G_{\partial \Omega_{23}}^{(1, \alpha)}\left(Z_{m}, X, t_{m \pm}\right)=(-1)^{\alpha} D g_{1}^{(\alpha)}\left(Z_{m}, X, t_{m \pm}\right)>0 \\
\text { for } Z_{m} \in \partial \Omega_{23} \text { in } \Omega_{\alpha}(\alpha \in\{2,3\}), \\
G_{\partial \Omega_{14}}^{(0, \alpha)}\left(Z_{m}, X, t_{m \pm}\right)=g_{1}^{(\alpha)}\left(Z_{m}, X, t_{m \pm}\right)=0
\end{gathered}
$$

$$
\begin{array}{r}
(-1)^{\alpha} G_{\partial \Omega_{14}}^{(1, \alpha)}\left(Z_{m}, X, t_{m \pm}\right)=(-1)^{\alpha} D g_{1}^{(\alpha)}\left(Z_{m}, X, t_{m \pm}\right)<0 \\
\text { for } Z_{m} \in \partial \Omega_{14} \text { in } \Omega_{\alpha}(\alpha \in\{1,4\}) .
\end{array}
$$

(iv) The onset of a sliding flow on the boundaries of $\partial \Omega_{12}, \partial \Omega_{34}, \partial \Omega_{23}$, and $\partial \Omega_{14}$ for the controlled system satisfies

$$
\begin{aligned}
& G_{\partial \Omega_{12}}^{(0,1)}\left(Z_{m}, X, t_{m-}\right)=g_{2}^{(1)}\left(Z_{m}, X, t_{m-}\right)<0 \\
& G_{\partial \Omega_{12}}^{(0,2)}\left(Z_{m}, X, t_{m \pm}\right)=g_{2}^{(2)}\left(Z_{m}, X, t_{m \pm}\right)=0 \\
& G_{\partial \Omega_{12}}^{(1,2)}\left(Z_{m}, X, t_{m \pm}\right)=D g_{2}^{(2)}\left(Z_{m}, X, t_{m \pm}\right)>0 \\
& \text { from } \Omega_{1} \text { to } \Omega_{12} \text {, } \\
& G_{\partial \Omega_{34}}^{(0,3)}\left(Z_{m}, X, t_{m-}\right)=g_{2}^{(3)}\left(Z_{m}, X, t_{m-}\right)>0 \\
& G_{\partial \Omega_{34}}^{(0,4)}\left(Z_{m}, X, t_{m \pm}\right)=g_{2}^{(4)}\left(Z_{m}, X, t_{m \pm}\right)=0 \\
& G_{\partial \Omega_{34}}^{(1,4)}\left(Z_{m}, X, t_{m \pm}\right)=D g_{2}^{(4)}\left(Z_{m}, X, t_{m \pm}\right)<0 \\
& \text { from } \Omega_{3} \text { to } \Omega_{34} \text {, } \\
& G_{\partial \Omega_{23}}^{(0,2)}\left(Z_{m}, X, t_{m-}\right)=g_{1}^{(2)}\left(Z_{m}, X, t_{m-}\right)<0 \\
& G_{\partial \Omega_{23}}^{(0,3)}\left(Z_{m}, X, t_{m \pm}\right)=g_{1}^{(3)}\left(Z_{m}, X, t_{m \pm}\right)=0 \\
& G_{\partial \Omega_{23}}^{(1,3)}\left(Z_{m}, X, t_{m \pm}\right)=D g_{1}^{(3)}\left(Z_{m}, X, t_{m \pm}\right)>0 \\
& \text { from } \Omega_{2} \text { to } \Omega_{23} \text {, } \\
& G_{\partial \Omega_{14}}^{(0,4)}\left(Z_{m}, X, t_{m \pm}\right)=g_{1}^{(4)}\left(Z_{m}, X, t_{m \pm}\right)>0 \\
& G_{\partial \Omega_{14}}^{(0,1)}\left(Z_{m}, X, t_{m \pm}\right)=g_{1}^{(1)}\left(Z_{m}, X, t_{m \pm}\right)=0 \\
& G_{\partial \Omega_{14}}^{(1,1)}\left(Z_{m}, X, t_{m \pm}\right)=D g_{1}^{(1)}\left(Z_{m}, X, t_{m \pm}\right)<0
\end{aligned}
$$

from $\Omega_{4}$ to $\Omega_{14}$.

(v) The vanishing of a sliding flow from the boundaries of $\partial \Omega_{12}, \partial \Omega_{34}, \partial \Omega_{23}$, and $\partial \Omega_{14}$ to a domain for the controlled system satisfies

$$
\begin{gathered}
(-1)^{\beta} G_{\partial \Omega_{12}}^{(0, \beta)}\left(Z_{m}, X, t_{m-}\right)=(-1)^{\beta} g_{2}^{(\beta)}\left(Z_{m}, X, t_{m-}\right)>0 \\
G_{\partial \Omega_{12}}^{(0, \alpha)}\left(Z_{m}, X, t_{m \mp}\right)=g_{2}^{(\alpha)}\left(Z_{m}, X, t_{m \mp}\right)=0 \\
(-1)^{\alpha} G_{\partial \Omega_{12}}^{(1, \alpha)}\left(Z_{m}, X, t_{m \mp}\right)=(-1)^{\alpha} D g_{2}^{(\alpha)}\left(Z_{m}, X, t_{m \mp}\right)<0 \\
\text { for } Z_{m} \in \partial \Omega_{12} ; \alpha, \beta \in\{1,2\}, \beta \neq \alpha \\
\text { from } \partial \Omega_{12} \longrightarrow \Omega_{\alpha} \\
(-1)^{\beta} G_{\partial \Omega_{34}}^{(0, \beta)}\left(Z_{m}, X, t_{m-}\right)=(-1)^{\beta} g_{2}^{(\beta)}\left(Z_{m}, X, t_{m-}\right)<0 \\
G_{\partial \Omega_{34}}^{(0, \alpha)}\left(Z_{m}, X, t_{m \mp}\right)=g_{2}^{(\alpha)}\left(Z_{m}, X, t_{m \mp}\right)=0
\end{gathered}
$$




$$
\begin{array}{r}
(-1)^{\alpha} G_{\partial \Omega_{34}}^{(1, \alpha)}\left(Z_{m}, X, t_{m \mp}\right)=(-1)^{\alpha} D g_{2}^{(\alpha)}\left(Z_{m}, X, t_{m \mp}\right)>0 \\
\text { for } Z_{m} \in \partial \Omega_{34} ; \alpha, \beta \in\{3,4\}, \beta \neq \alpha \\
\text { from } \partial \Omega_{34} \longrightarrow \Omega_{\alpha}, \\
(-1)^{\beta} G_{\partial \Omega_{23}}^{(0, \beta)}\left(Z_{m}, X, t_{m-}\right)=(-1)^{\beta} g_{1}^{(\beta)}\left(Z_{m}, X, t_{m-}\right)<0 \\
G_{\partial \Omega_{23}}^{(0, \alpha)}\left(Z_{m}, X, t_{m \mp}\right)=g_{1}^{(\alpha)}\left(Z_{m}, X, t_{m \mp}\right)=0 \\
(-1)^{\alpha} G_{\partial \Omega_{23}}^{(1, \alpha)}\left(Z_{m}, X, t_{m \mp}\right)=(-1)^{\alpha} D g_{1}^{(\alpha)}\left(Z_{m}, X, t_{m \mp}\right)>0 \\
\text { for } Z_{m} \in \partial \Omega_{23} ; \alpha, \beta \in\{2,3\}, \beta \neq \alpha \\
\quad \text { from } \partial \Omega_{23} \longrightarrow \Omega_{\alpha} \\
(-1)^{\beta} G_{\partial \Omega_{14}}^{(0, \beta)}\left(Z_{m}, X, t_{m-}\right)=(-1)^{\beta} g_{1}^{(\beta)}\left(Z_{m}, X, t_{m-}\right)>0 \\
G_{\partial \Omega_{14}}^{(0, \alpha)}\left(Z_{m}, X, t_{m \mp}\right)=g_{1}^{(\alpha)}\left(Z_{m}, X, t_{m \mp}\right)=0 \\
(-1)^{\alpha} G_{\partial \Omega_{14}}^{(1, \alpha)}\left(Z_{m}, X, t_{m \mp}\right)=(-1)^{\alpha} D g_{1}^{(\alpha)}\left(Z_{m}, X, t_{m \mp}\right)<0 \\
\text { for } Z_{m} \in \partial \Omega_{14} ; \alpha, \beta \in\{1,4\}, \beta \neq \alpha \\
\text { from } \partial \Omega_{14} \longrightarrow \Omega \Omega_{\alpha} .
\end{array}
$$

4.2. Synchronization Conditions. With the theory of the switchability of a flow, the conditions for the synchronization of the two dynamical systems at the intersection of the two separation boundaries $\left(Z_{m}=0\right)$ are

$$
\begin{aligned}
& G_{\partial \Omega_{14}}^{(1)}\left(Z_{m}, X, t_{m-}\right)=g_{1}^{(1)}\left(Z_{m}, X, t_{m-}\right)<0 \\
& G_{\partial \Omega_{12}}^{(1)}\left(Z_{m}, X, t_{m-}\right)=g_{2}^{(1)}\left(Z_{m}, X, t_{m-}\right)<0 \\
& \text { for } Z_{m} \in \partial \Omega_{12} \cap \partial \Omega_{14} \text { on } \Omega_{1}, \\
& G_{\partial \Omega_{12}}^{(2)}\left(Z_{m}, X, t_{m-}\right)=g_{2}^{(2)}\left(Z_{m}, X, t_{m-}\right)>0 \\
& G_{\partial \Omega_{23}}^{(2)}\left(Z_{m}, X, t_{m-}\right)=g_{1}^{(2)}\left(Z_{m}, X, t_{m-}\right)<0 \\
& \text { for } Z_{m} \in \partial \Omega_{12} \cap \partial \Omega_{23} \text { on } \Omega_{2}, \\
& G_{\partial \Omega_{23}}^{(3)}\left(Z_{m}, X, t_{m-}\right)=g_{1}^{(3)}\left(Z_{m}, X, t_{m-}\right)>0 \\
& G_{\partial \Omega_{34}}^{(3)}\left(Z_{m}, X, t_{m-}\right)=g_{2}^{(3)}\left(Z_{m}, X, t_{m-}\right)>0 \\
& \text { for } Z_{m} \in \partial \Omega_{23} \cap \partial \Omega_{34} \text { on } \Omega_{3}, \\
& G_{\partial \Omega_{34}}^{(4)}\left(Z_{m}, X, t_{m-}\right)=g_{2}^{(4)}\left(Z_{m}, X, t_{m-}\right)<0 \\
& G_{\partial \Omega_{41}}^{(4)}\left(Z_{m}, X, t_{m-}\right)=g_{1}^{(4)}\left(Z_{m}, X, t_{m-}\right)>0 \\
& \text { for } Z_{m} \in \partial \Omega_{34} \cap \partial \Omega_{14} \text { on } \Omega_{4} .
\end{aligned}
$$

From (27), we define four basic functions:

$$
\begin{aligned}
& g_{1}\left(Z_{m}, X, t\right) \equiv z_{2}^{(\alpha)}-k_{1} \quad \text { in } \Omega_{\alpha} \text { for } \alpha=1,2, \\
& g_{2}\left(Z_{m}, X, t\right) \equiv z_{2}^{(\alpha)}+k_{1} \quad \text { in } \Omega_{\alpha} \text { for } \alpha=3,4,
\end{aligned}
$$

$$
\begin{aligned}
& g_{3}\left(Z_{m}, X, t\right)=\mathscr{G}\left(Z^{(\alpha)}, X, t\right)-k_{2} \text { in } \Omega_{\alpha} \text { for } \alpha=1,4, \\
& g_{4}\left(Z_{m}, X, t\right)=\mathscr{G}\left(Z^{(\alpha)}, X, t\right)+k_{2} \quad \text { in } \Omega_{\alpha} \text { for } \alpha=2,3 .
\end{aligned}
$$

The synchronization conditions in (41) become

$$
\begin{gathered}
g_{1}\left(Z_{m}, X, t_{m-}\right) \equiv z_{2 m}-k_{1}<0, \\
g_{2}\left(Z_{m}, X, t_{m-}\right) \equiv z_{2 m}+k_{1}>0, \\
g_{3}\left(Z_{m}, X, t_{m-}\right)=\mathscr{G}\left(Z_{m}, X, t_{m-}\right)-k_{2}<0, \\
g_{4}\left(Z_{m}, X, t_{m-}\right)=\mathscr{G}\left(Z_{m}, X, t_{m-}\right)+k_{2}>0 .
\end{gathered}
$$

Let $Z_{m}^{(\alpha)}=Z_{m}=0$. The conditions are

$$
\begin{gathered}
g_{1}\left(Z_{m}, X, t_{m-}\right) \equiv-k_{1}<0, \\
g_{2}\left(Z_{m}, X, t_{m-}\right) \equiv k_{1}>0, \\
g_{3}\left(Z_{m}, X, t_{m-}\right)=\mathscr{G}\left(0, X, t_{m-}\right)-k_{2}<0, \\
g_{4}\left(Z_{m}, X, t_{m-}\right)=\mathscr{G}\left(0, X, t_{m-}\right)+k_{2}>0,
\end{gathered}
$$

where the $\mathscr{G}$-function becomes

$$
\begin{aligned}
\mathscr{G}\left(Z^{(\alpha)}, X, t\right)= & -\varepsilon\left[\sin ^{2} x_{1}-1\right] x_{2} \cos x_{1}-\sin x_{1} \\
& +a_{0} \sin x_{1} \cos x_{1} \\
& -A_{0} \cos \omega t \cos x_{1}+x_{2}^{2} \sin x_{1}
\end{aligned}
$$

If $k_{1}>0, k_{2}>0$, the first two equations can be satisfied, and the last two equations give the synchronization invariant set; that is,

$$
-k_{2}<\mathscr{G}\left(0, X, t_{m-}\right)<k_{2}
$$

Consider a small neighborhood of $Z_{m}=0$; the attractive conditions for $\left|Z-Z_{m}\right|<\varepsilon$ are given by

$$
\begin{aligned}
& 0 \leq z_{2}<k_{1}, \quad \mathscr{G}(Z, X, t)<k_{2} \text { for } z_{1} \in[0,+\infty) \text { in } \Omega_{1} \text {, } \\
& 0 \leq z_{2}<k_{1}, \quad-k_{2}<\mathscr{G}(Z, X, t) \quad \text { for } z_{1} \in[0,+\infty) \text { in } \Omega_{2} \text {, } \\
& -k_{1}<z_{2} \leq 0, \quad-k_{2}<\mathscr{G}(Z, X, t) \\
& \text { for } z_{1} \in(-\infty, 0] \text { in } \Omega_{3} \text {, } \\
& -k_{1}<z_{2} \leq 0, \quad \mathscr{G}(Z, X, t)<k_{2} \quad \text { for } z_{1} \in(-\infty, 0] \text { in } \Omega_{4} \text {. }
\end{aligned}
$$

From the foregoing equation, the initial point $z_{1}^{*}$ and $z_{2}^{*}$ can be obtained for the system in relative coordinate. Thus the initial conditions for the controlled slave system should be determined by

$$
y_{1}=z_{1}^{*}+\sin x_{1}, \quad y_{2}=z_{2}^{*}+x_{2} \cos x_{1} .
$$


The conditions of synchronization vanishing for the controlled slave system with $Z^{(\alpha)}\left(t_{\mp}\right)=Z_{m}^{(\alpha)}=\left(z_{1 m}^{(\alpha)}, z_{2 m}^{(\alpha)}\right)=$ $Z_{m}$ are

$$
\begin{gathered}
g_{1}\left(Z_{m}^{(\alpha)}, X, t_{\mp}\right)=z_{2 m}^{(\alpha)}-k_{1}=0, \\
D g_{1}\left(Z_{m}^{(\alpha)}, X, t_{m \mp}\right)=\mathscr{G}\left(Z_{m}^{(\alpha)}, X, t_{m \mp}\right)>0, \\
g_{2}\left(Z_{m}^{(\beta)}, X, t_{m-}\right)=z_{2 m}^{(\beta)}+k_{1}>0 ; \\
\text { for }(\alpha, \beta)=\{(1,4),(2,3)\},
\end{gathered}
$$

from $Z_{m+\varepsilon}=y_{1}-\sin x_{1}>0$; and

$$
\begin{gathered}
g_{1}\left(Z_{m}^{(\alpha)}, X, t_{m-}\right)=z_{2 m}^{(\alpha)}-k_{1}<0, \\
g_{2}\left(Z_{m}^{(\beta)}, X, t_{m \mp}\right)=z_{2 m}^{(\beta)}+k_{1}=0, \\
D g_{2}\left(Z_{m}^{(\beta)}, X, t_{m \mp}\right)=\mathscr{G}\left(Z_{m}^{(\beta)}, X, t_{m \mp}\right)<0 ; \\
\text { for }(\alpha, \beta)=\{(1,4),(2,3)\},
\end{gathered}
$$

from $Z_{m+\varepsilon}=y_{1}-\sin x_{1}<0$; and

$$
\begin{array}{r}
g_{3}\left(Z_{m}^{(\alpha)}, X, t_{m \mp}\right)=\mathscr{G}\left(Z_{m}^{(\alpha)}, X, t_{m \mp}\right)-k_{2}=0, \\
D g_{3}\left(Z_{m}^{(\alpha)}, X, t_{m \mp}\right)=D \mathscr{G}\left(Z_{m}^{(\alpha)}, X, t_{m \mp}\right)>0, \\
g_{4}\left(Z_{m}^{(\beta)}, X, t_{m-}\right)=\mathscr{G}\left(Z_{m}^{(\beta)}, X, t_{m-}\right)+k_{2}>0 ; \\
\text { for }(\alpha, \beta)=\{(1,2),(4,3)\},
\end{array}
$$

from $\dot{Z}_{m+\varepsilon}=y_{2}-x_{2} \cos x_{1}>0$; and

$$
\begin{array}{r}
g_{3}\left(Z_{m}^{(\alpha)}, X, t_{m-}\right)=\mathscr{G}\left(Z_{m}^{(\alpha)}, X, t_{m-}\right)-k_{2}<0, \\
g_{4}\left(Z_{m}^{(\beta)}, X, t_{m \mp}\right)=\mathscr{G}\left(Z_{m}^{(\beta)}, X, t_{m \mp}\right)+k_{2}=0, \\
D g_{4}\left(Z_{m}^{(\beta)}, X, t_{m \mp}\right)=D \mathscr{G}\left(Z_{m}^{(\beta)}, X, t_{m \mp}\right)<0 ; \\
\text { for }(\alpha, \beta)=\{(1,2),(4,3)\},
\end{array}
$$

from $\dot{Z}_{m+\varepsilon}=y_{2}-x_{2} \cos x_{1}<0$.

The conditions for onset of synchronization for the controlled slave system with $Z^{(\alpha)}\left(t_{\bar{\mp}}\right)=Z_{m}^{(\alpha)}=Z_{m}$ are

$$
\begin{gathered}
g_{1}\left(Z_{m}^{(\alpha)}, X, t_{m \pm}\right)=z_{2 m}^{(\alpha)}-k_{1}=0, \\
D g_{1}\left(Z_{m}^{(\alpha)}, X, t_{m \pm}\right)=\mathscr{G}\left(Z_{m}^{(\alpha)}, X, t_{m \pm}\right)>0, \\
g_{2}\left(Z_{m}^{(\beta)}, X, t_{m-}\right)=z_{2 m}^{(\beta)}+k_{1}>0 ; \\
\text { for }(\alpha, \beta)=\{(1,4),(2,3)\},
\end{gathered}
$$

from $Z_{m-\varepsilon}=y_{1}-\sin x_{1}>0$; and

$$
\begin{gathered}
g_{1}\left(Z_{m}^{(\alpha)}, X, t_{m-}\right)=z_{2 m}^{(\alpha)}-k_{1}<0, \\
g_{2}\left(Z_{m}^{(\beta)}, X, t_{m \pm}\right)=z_{2 m}^{(\beta)}+k_{1}=0, \\
D g_{2}\left(Z_{m}^{(\beta)}, X, t_{m \pm}\right)=\mathscr{G}\left(Z_{m}^{(\beta)}, X, t_{m \pm}\right)<0 ; \\
\text { for }(\alpha, \beta)=\{(1,4),(2,3)\},
\end{gathered}
$$

from $Z_{m-\varepsilon}=y_{1}-\sin x_{1}<0$; and

$$
\begin{array}{r}
g_{3}\left(Z_{m}^{(\alpha)}, X, t_{m \pm}\right)=\mathscr{G}\left(Z_{m}^{(\alpha)}, X, t_{m \pm}\right)-k_{2}=0, \\
D g_{3}\left(Z_{m}^{(\alpha)}, X, t_{m \pm}\right)=D \mathscr{G}\left(Z_{m}^{(\alpha)}, X, t_{m \pm}\right)>0, \\
g_{4}\left(Z_{m}^{(\beta)}, X, t_{m-}\right)=\mathscr{G}\left(Z_{m}^{(\beta)}, X, t_{m-}\right)+k_{2}>0 ; \\
\text { for }(\alpha, \beta)=\{(1,2),(4,3)\},
\end{array}
$$

from $\dot{Z}_{m-\varepsilon}=y_{2}-x_{2} \cos x_{1}>0$; and

$$
\begin{array}{r}
g_{3}\left(Z_{m}^{(\alpha)}, X, t_{m-}\right)=\mathscr{G}\left(Z_{m}^{(\alpha)}, X, t_{m-}\right)-k_{2}<0, \\
g_{4}\left(Z_{m}^{(\beta)}, X, t_{m \pm}\right)=\mathscr{G}\left(Z_{m}^{(\beta)}, X, t_{m \pm}\right)+k_{2}=0, \\
D g_{4}\left(Z_{m}^{(\beta)}, X, t_{m \pm}\right)=D \mathscr{G}\left(Z_{m}^{(\beta)}, X, t_{m \pm}\right)<0 ; \\
\text { for }(\alpha, \beta)=\{(1,2),(4,3)\},
\end{array}
$$

from $\dot{Z}_{m-\varepsilon}=y_{2}-x_{2} \cos x_{1}<0$.

\section{Conflict of Interests}

The authors declare that there is no conflict of interests regarding the publication of this paper.

\section{Acknowledgments}

This work was supported by the National Natural Science Foundation of China (11171192) and the Specialized Research Fund for the Doctoral Program of Higher Education of China (20123704110001).

\section{References}

[1] C. Huygens, Horologium Oscillatorium, Apud F. Muguet, Paris, France, 1673, English translation: The Pendulum Clock, Iowa State University, Ames, Iowa, USA, 1986.

[2] G. Leonov, H. Nijmeijer, A. Pogromsky, and A. Fradkov, Dynamics and Control of Hybrid Mechanical Systems, World Scientific, Singapore, 2010.

[3] S. Boccaletti, The Synchronized Dynamics of Complex Systems, vol. 6 of Monograph Series on Nonlinear Science and Complexity, Elsevier, Amsterdam, The Netherlands, 2008.

[4] A. Pikovsky, M. Rosenblum, and J. Kurths, Synchronization: A Universal Concept in Nonlinear Sciences, vol. 12 of Cambridge Nonlinear Science Series, Cambridge University Press, Cambridge, UK, 2001.

[5] L. M. Pecora and T. L. Carroll, "Synchronization in chaotic systems," Physical Review Letters, vol. 64, no. 8, pp. 821-824, 1990.

[6] T. L. Carroll and L. M. Pecora, "Synchronizing chaotic circuits," IEEE Transactions on Circuits and Systems, vol. 38, no. 4, pp. 453-456, 1991.

[7] K. Pyragas, "Continuous control of chaos by self-controlling feedback," Physics Letters A, vol. 170, no. 6, pp. 421-428, 1992. 
[8] T. Kapitaniak, "Synchronization of chaos using continuous control," Physical Review E: Statistical Physics, Plasmas, Fluids, and Related Interdisciplinary Topics, vol. 50, no. 2, pp. $1642-$ 1644, 1994.

[9] M. Ding and E. Ott, "Enhancing synchronism of chaotic systems," Physical Review E: Statistical Physics, Plasmas, Fluids, and Related Interdisciplinary Topics, vol. 49, no. 2, pp. R945R948, 1994.

[10] N. F. Rulkov, M. M. Sushchik, L. S. Tsimring, and H. D. I. Abarbanel, "Generalized synchronization of chaos in directionally coupled chaotic systems," Physical Review E: Statistical Physics, Plasmas, Fluids, and Related Interdisciplinary Topics, vol. 51, no. 2, pp. 980-994, 1995.

[11] L. Kocarev and U. Parlitz, "General approach for chaotic synchronization with applications to communication," Physical Review Letters, vol. 74, no. 25, pp. 5028-5031, 1995.

[12] K. Pyragas, "Weak and strong synchronization of chaos," Physical Review E: Statistical Physics, Plasmas, Fluids, and Related Interdisciplinary Topics, vol. 54, no. 5, pp. R4508-R4511, 1996.

[13] M. Ding, E.-J. Ding, W. L. Ditto et al., "Control and synchronization of chaos in high-dimensional systems: review of some recent results," Chaos, vol. 7, no. 4, pp. 644-652, 1997.

[14] E. Campos, J. Urías, and N. F. Rulkov, "Multimodal synchronization of chaos," Chaos, vol. 14, no. 1, pp. 48-54, 2004.

[15] A. Teufel, A. Steindl, and H. Troger, "Synchronization of two flow excited pendula," Communications in Nonlinear Science and Numerical Simulation, vol. 11, no. 5, pp. 577-594, 2006.

[16] Y. Chen, G. Rangarajan, and M. Ding, "Stability of synchronized dynamics and pattern formation in coupled systems: review of some recent results," Communications in Nonlinear Science and Numerical Simulation, vol. 11, no. 8, pp. 934-960, 2006.

[17] Z. Chen, "Complete synchronization for impulsive CohenGrossberg neural networks with delay under noise perturbation," Chaos, Solitons and Fractals, vol. 42, no. 3, pp. 1664-1669, 2009.

[18] Z. Chen, W. Lin, and J. Zhou, "Complete and generalized synchronization in a class of noise perturbed chaotic systems," Chaos, vol. 17, no. 2, Article ID 681605, 8 pages, 2007.

[19] A. C. J. Luo, "A theory for non-smooth dynamic systems on the connectable domains," Communications in Nonlinear Science and Numerical Simulation, vol. 10, no. 1, pp. 1-55, 2005.

[20] A. C. J. Luo, Singularity and Dynamics on Discontinuous Vector Fields, vol. 3 of Monograph Series on Nonlinear Science and Complexity, Elsevier, Amsterdam, The Netherlands, 2006.

[21] A. C. J. Luo, Global Transversality, Resonance and Chaotic Dynamics, World Scientific, River Edge, NJ, USA, 2008.

[22] A. C. J. Luo, "A theory for flow switchability in discontinuous dynamical systems," Nonlinear Analysis: Hybrid Systems, vol. 2, no. 4, pp. 1030-1061, 2008.

[23] A. C. J. Luo, Discontinuous Dynamical Systems on Time-Varying Domains, HEP-Springer, Dordrecht, The Netherlands, 2009.

[24] A. C. J. Luo and F. Min, "Synchronization of a periodically forced Duffing oscillator with a periodically excited pendulum," Nonlinear Analysis: Real World Applications, vol. 12, no. 3, pp. 1810-1827, 2011. 


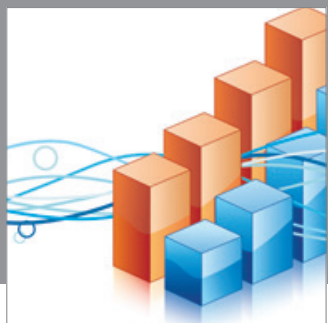

Advances in

Operations Research

mansans

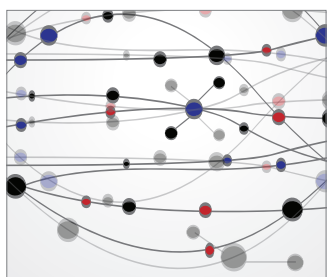

The Scientific World Journal
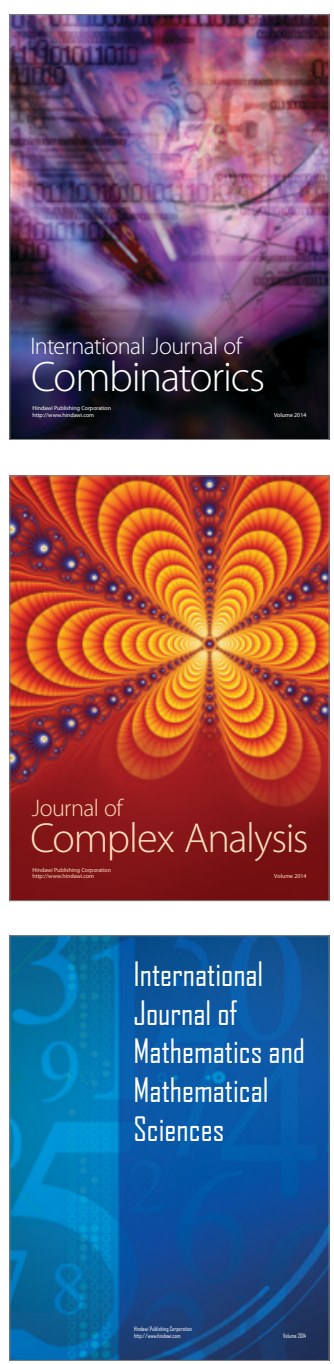
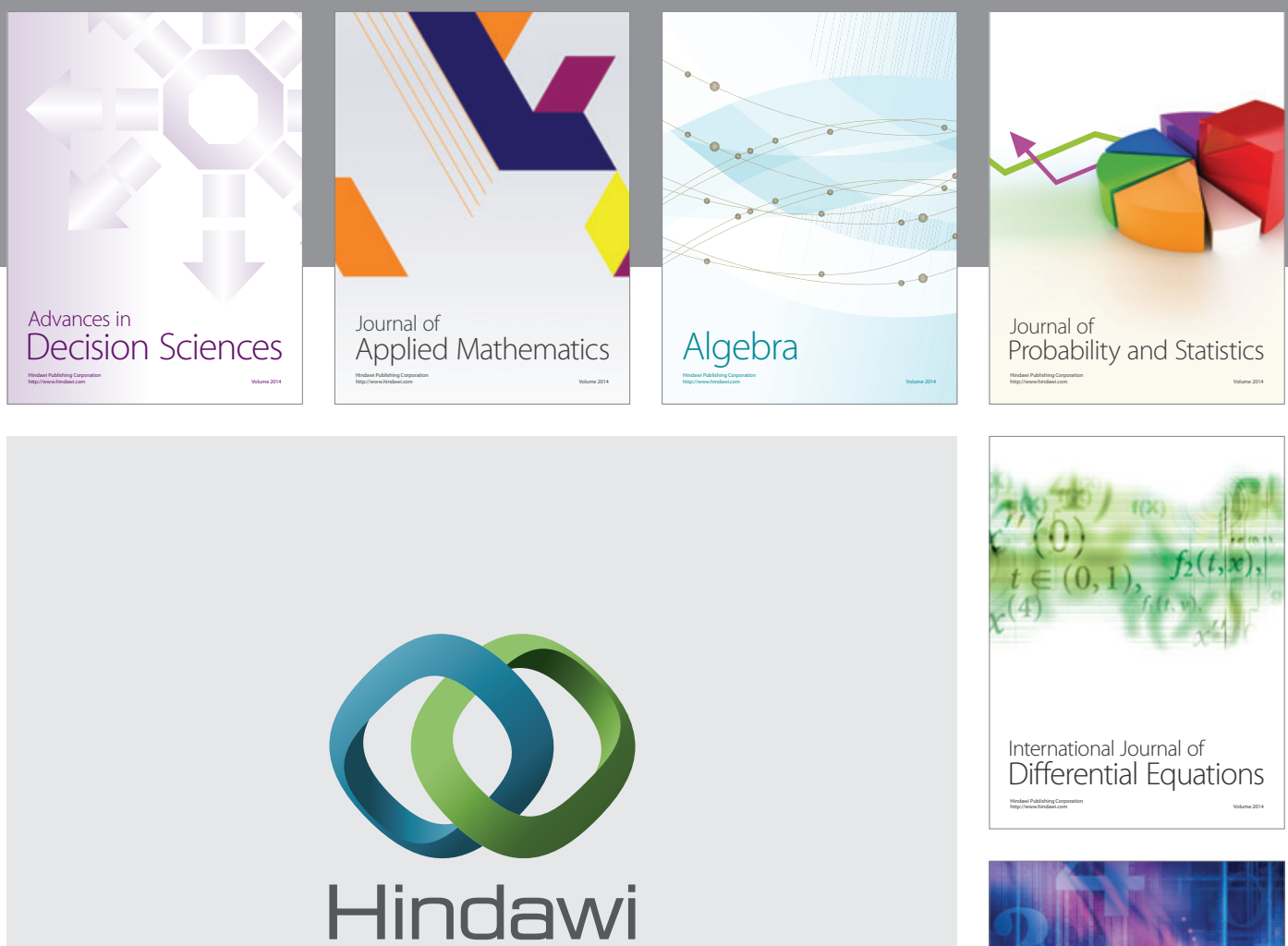

Submit your manuscripts at http://www.hindawi.com
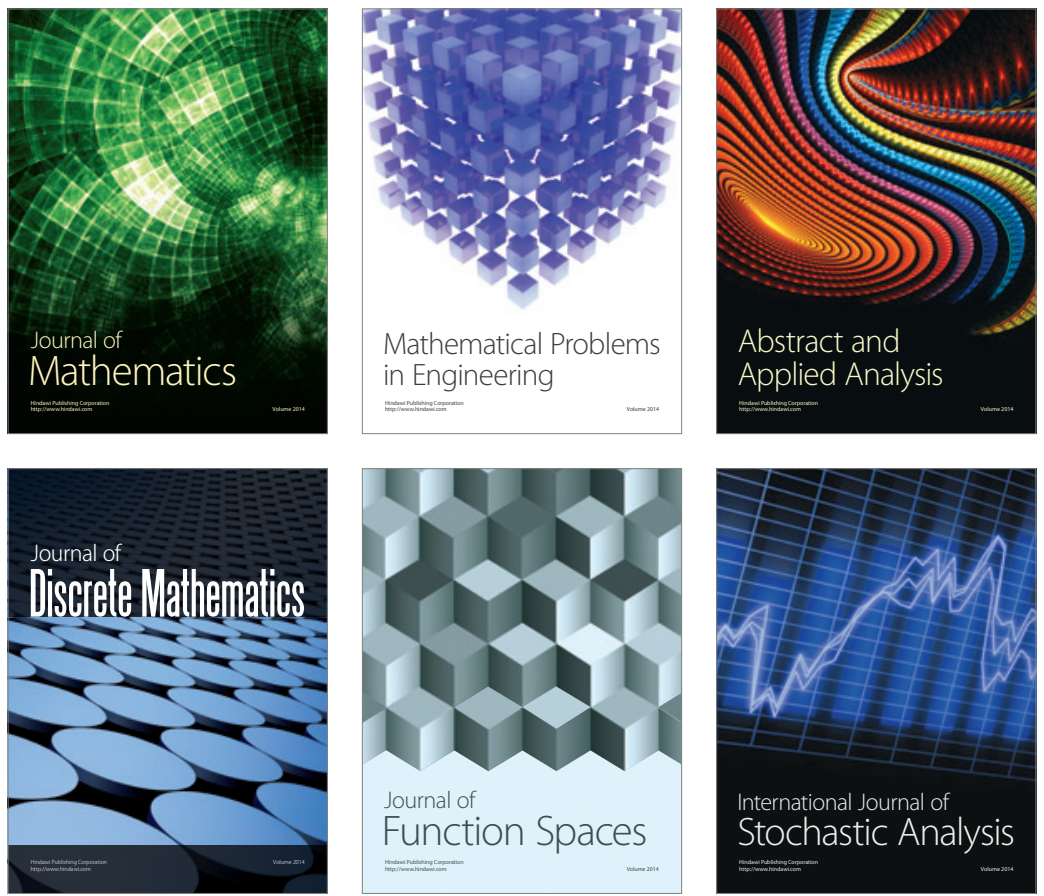

Journal of

Function Spaces

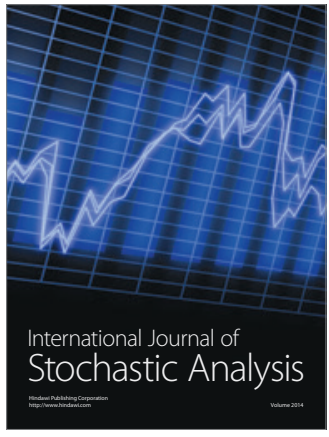

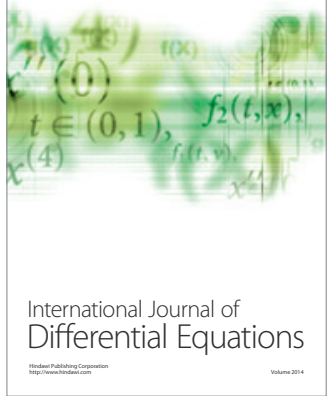
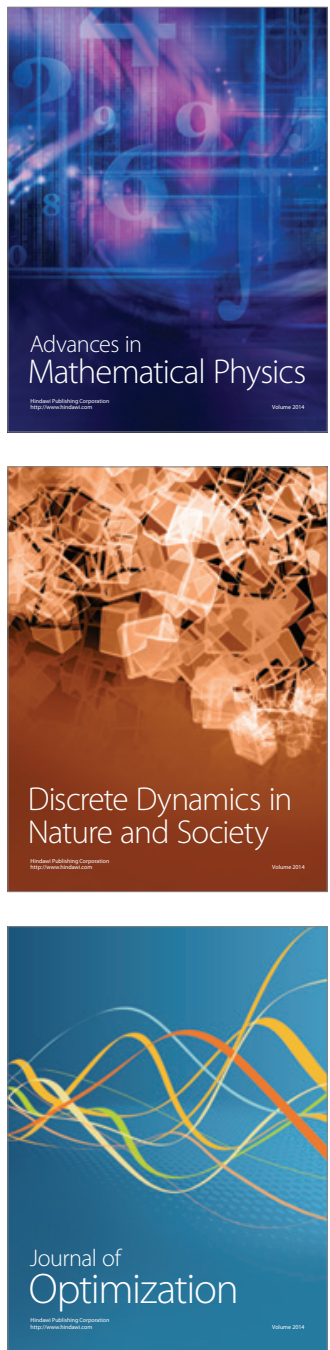